\title{
Near and intermediate fields of an ultrashort pulse transmitted through Young's double-slit experiment
}

\author{
A. M. Nugrowati, ${ }^{1, *}$ S. F. Pereira, ${ }^{1}$ and A. S. van de Nes ${ }^{2, \dagger}$ \\ ${ }^{1}$ Optics Research Group, Department of Imaging Science and Technology, Delft University of Technology, Lorentzweg 1, \\ 2628 CJ Delft, The Netherlands \\ ${ }^{2}$ Department of Physics, Imperial College London, Prince Consort Road, London SW7 2BW, United Kingdom
}

(Received 25 January 2008; published 12 May 2008)

\begin{abstract}
We present a systematic study of the transmitted field of an ultrashort pulse through Young's double slit. We show how the spatial-temporal distribution of the field in the near and intermediate zone is affected by the input polarization state of the pulse. The analysis has been separated to study the dispersion, the diffraction and the interference effects individually. A combination of these effects provide the key to understand the temporal dependence of the field observed at various distinctive locations. A thorough understanding of the near- and intermediate-field distribution of a single- or double-slit for an ultrashort pulse can be exploited in many near-field applications, and tailored by tuning the width, thickness, and separation of the slits.
\end{abstract}

DOI: 10.1103/PhysRevA.77.053810

PACS number(s): 42.25.Fx, 42.65.Re, 42.25.Ja, 42.25.Hz

\section{INTRODUCTION}

Ultrashort pulses find application in, for example, telecommunications, micromachining, and terahertz generation, and are used in research to advance the study in fields such as femtochemistry, multiphoton fluorescence microscopy, and spectroscopy. The short duration and high intensity of the ultrashort pulse allows for the use of nonlinear effects in light-matter interaction, improving the sensitivity of a whole range of experiments in far and near field optics [1] and, in particular, in optical microscopy [2]. Several experiments to generate or detect ultrashort pulses have been reported in quick succession at different wavelength ranges $[3,4]$. However, fundamental physical phenomena, such as the diffraction and interference of the ultrashort pulse by simple structures are not straightforward to understand and, when the structure is of wavelength dimensions, require a rigorous treatment.

Earlier calculations of diffraction patterns by an ultrashort pulse have used scalar theory to provide analytical or numerical solutions [5-10]. They have shown that the diffraction pattern of an aperture observed at a plane perpendicular to the propagation axis has a different time dependence than that of the incident pulse, demonstrating that the spectral content varies within the spatial domain. This effect can be explained in terms of Huygens waves originating from different locations in the exit plane of the aperture, resulting in a difference in optical path length at the observation plane. However, such a scalar approach is not valid in the near and intermediate field and neither does it treat the different polarization states properly.

Moreover, light passing through small apertures in a metal layer has attracted a lot of attention in the last decade [11]. The broad spectral range of an ultrashort pulse that illuminates such small structures yields a unique diffraction pattern that depends on the combined effects of the aperture geom-

\footnotetext{
*a.m.nugrowati@tudelft.nl

† arthur.vandenes@imperial.ac.uk
}

etry, the bandwidth of the pulse, the polarization state of the illumination, and the material dispersion. Given the complexity of the problem, one cannot avoid the use of numerical methods to obtain a rigorous solution. Most commonly, a finite-difference time-domain (FDTD) method is chosen $[12,13]$, since it operates in the time domain. However, frequency domain methods have also been used [14-16] to calculate ultrashort pulse propagation and diffraction. When a modal decomposition technique is used, additional information can be obtained to identify specific characteristics of the propagation of pulsed light through small apertures.

In this paper, we systematically analyze the propagation of a linearly polarized ultrashort pulse, as has been used in experiments [17], initially through a single slit and afterwards through Young's double-slit in an aluminum layer. The single slit allows us to study the dispersion and diffraction effects, while the addition of another slit forces us to consider possible interference effects. We apply a fully vectorial frequency domain method for the calculation of the diffracted fields. Analysis of the temporal and spatial evolution of the diffraction pattern is performed for both the transverse electric (TE) and magnetic (TM) polarization state in the near and intermediate zone. Specifications of the optical configuration, including the model for the ultrashort pulse, the material refractive index, and the calculation tool are described in Sec. II. The diffraction of the ultrashort pulse by the slit structures is presented and analyzed in Sec. III, followed by conclusions in Sec. IV.

\section{SPECIFICATION OF THE OPTICAL CONFIGURATION}

\section{A. Ultrashort pulse illumination}

Most representations of the spectral distribution of the ultrashort pulse consist of a Gaussian spectrum that includes negative frequencies. However, this leads to noncausal artifacts in the time response. Therefore, we apply an analytic model which tends to a Gaussian distribution but excludes negative frequencies $[18,19]$. By introducing a nonzero cutoff frequency as described in Ref. [9], we obtain an ul- 

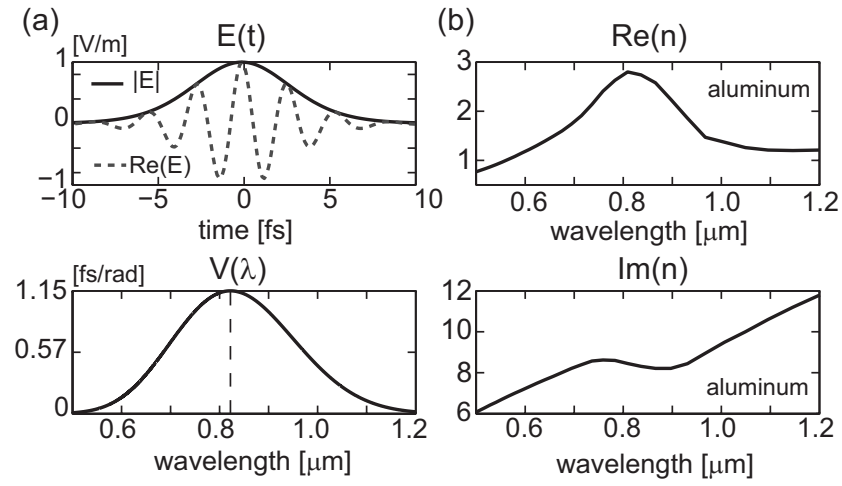

FIG. 1. (a) The time trace (top) of the pulse consisting of five cycles within a time span of $7.5 \mathrm{fs}$ and the corresponding spectral distribution (bottom), with the peak at $825 \mathrm{~nm}$. (b) The refractive index of aluminum as a function of the wavelength, separated in the real (top) and the imaginary component (bottom).

trashort pulse that extends from visible to near infrared wavelengths, similar as used in experiments reported by Ref. [17]. The expression for this spectral distribution is given by

$$
V(\omega)=\left(\frac{s+1}{\omega_{1}}\right)^{s+1} \frac{\left(\omega-\omega_{0}\right)^{s}}{\Gamma(s+1)} \exp \left[-(s+1) \frac{\omega-\omega_{0}}{\omega_{1}}\right],
$$

when $\omega \geq \omega_{0}$ and $V(\omega)=0$ otherwise. We choose a cutoff frequency of $\omega_{0}=1.2 \mathrm{rad} / \mathrm{fs}$, the spectral bandwidth is determined by $\omega_{1}=1.2 \mathrm{rad} / \mathrm{fs}$, and the positive integer $s=10$ is proportional to the number of cycles in the pulse. Based on these parameters, the ultrashort pulse contains five cycles, has a total length of $7.5 \mathrm{fs}$, a bandwidth of $0.5<\lambda$ $<1.2 \mu \mathrm{m}$, with the peak at $\lambda_{c}=825 \mathrm{~nm}$. In Fig. 1(a), we show the time trace (top) and corresponding wavelength distribution (bottom) of the ultrashort pulse.

\section{B. Slit structures}

We consider consecutively a single- and a double-slit structure in an aluminum layer surrounded by air, in order to study first the dispersion and diffraction effects by an aperture and next the interference effects due to the presence of a second aperture. The optical properties of aluminum for the bandwidth of interest exhibits a dynamic response as a function of the wavelength, shown in Fig. 1(b) for the real (top) and imaginary part (bottom) of the refractive index [20]. The metal layer containing the slit structures extends to infinity along the $x$ and $y$ directions, and is illuminated by a planar ultrashort pulse propagating along the $z$ axis. The slit structures are defined by the metal layer thickness $d$, the width of the slits $w$, and the distance between the two slits $p$ for the double-slit structure. A schematic overview of the structures is given in Fig. 2(a) for the single and in Fig. 2(b) for the double slit. We choose a metal thickness and slit width of $d=w=0.7 \mu \mathrm{m}$. Therefore, the aperture is subwavelength for $\lambda=1.2 \mu \mathrm{m}$ and larger than a wavelength for $\lambda=0.5 \mu \mathrm{m}$, the outer limits of the pulse spectrum shown in Fig. 1(a). The slit separation is chosen to be $p=3.5 \mu \mathrm{m}$. In this way, the diffracted field right behind each of the slits is initially well separated from the other when the peak of the pulse reaches the slit entrance. (a)

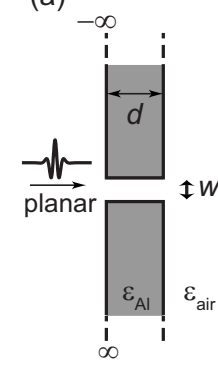

(b)

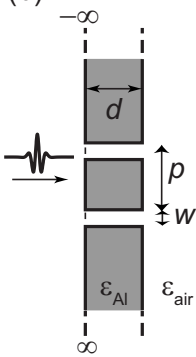

(c)

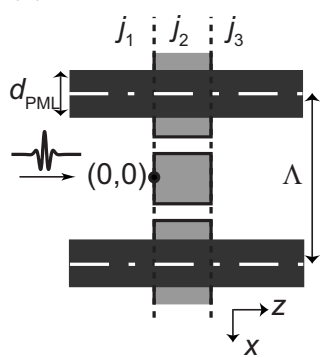

FIG. 2. Geometry of (a) a single- and (b) a double-slit structure in an aluminum layer. The structure is illuminated by a planar ultrashort pulse at normal incidence. (c) Schematic of the numerical representation. The double-slit structure is represented by a threelayer system with a periodicity $\Lambda$. Perfectly matched layers and a nonlinear coordinate transformation are applied in the dark gray areas of thickness $d_{\mathrm{PML}}$ in order to prevent cross talk between the cells (in between two dash dotted lines).

\section{Calculation method}

The frequency domain method used in this paper is known as the Fourier modal method [21], widely applied for calculating the electromagnetic field of a structure with a periodic boundary, and selected because of its versatility and clear physical interpretation. In essence, a slit configuration has a two-dimensional geometry. We assume a constant magnetic permeability $\mu_{0}$. The electric permittivity $\epsilon$ is separated in piecewise constant regions along the $z$ axis, where each region is treated as an individual layer. An example of the representation for a double-slit structure is given in Fig. 2(c). The electric permittivity function in each layer is expanded in Fourier modes for variations along the $x$ direction, assuming a repetition of the structure with period $\Lambda$. To be able to simulate the desired isolated structure, the method allows for a perfectly matched layer and nonlinear coordinate transformation [22]. Following the notation of Ref. [22], we choose $f_{\mathrm{ct}}=(1+i) / 2$ for the coordinate transformation to $f\left(x^{\prime}\right)$, and $f_{\mathrm{PML}}=(1-i) / 2$ for the perfectly matched layer. Further details about the specific implementation of the method can be found in Ref. [23] (Sec. MM1).

To simulate the time dependence of an ultrashort pulse using the frequency domain method, we have discretized the wavelength distribution into a hundred equidistant steps separated by $\Delta \lambda$. For each wavelength, we calculate the electromagnetic field distribution in the region of interest with the method described above. The evolution of the field in the time domain is then obtained by recombining the individual field distributions $U(\lambda ; x, z)$, as expressed by

$$
U(t ; x, z)=\sum_{\lambda} U(\lambda ; x, z) V(\lambda) \exp \left[-i \frac{2 \pi c}{\lambda} t\right] \frac{2 \pi c \Delta \lambda}{\lambda^{2}} .
$$

The origin of the pulse is chosen such that the center reaches the slit at $z=0$ when $t=0$ fs. The incident pulse is of normal incidence with respect to the aluminum layer. The thickness of the perfectly matched layers $d_{\mathrm{PML}}=0.3 \mu \mathrm{m}$ in combination with a nonlinear coordinate transformation in the same region is enough to remove any parasitic reflections from the 

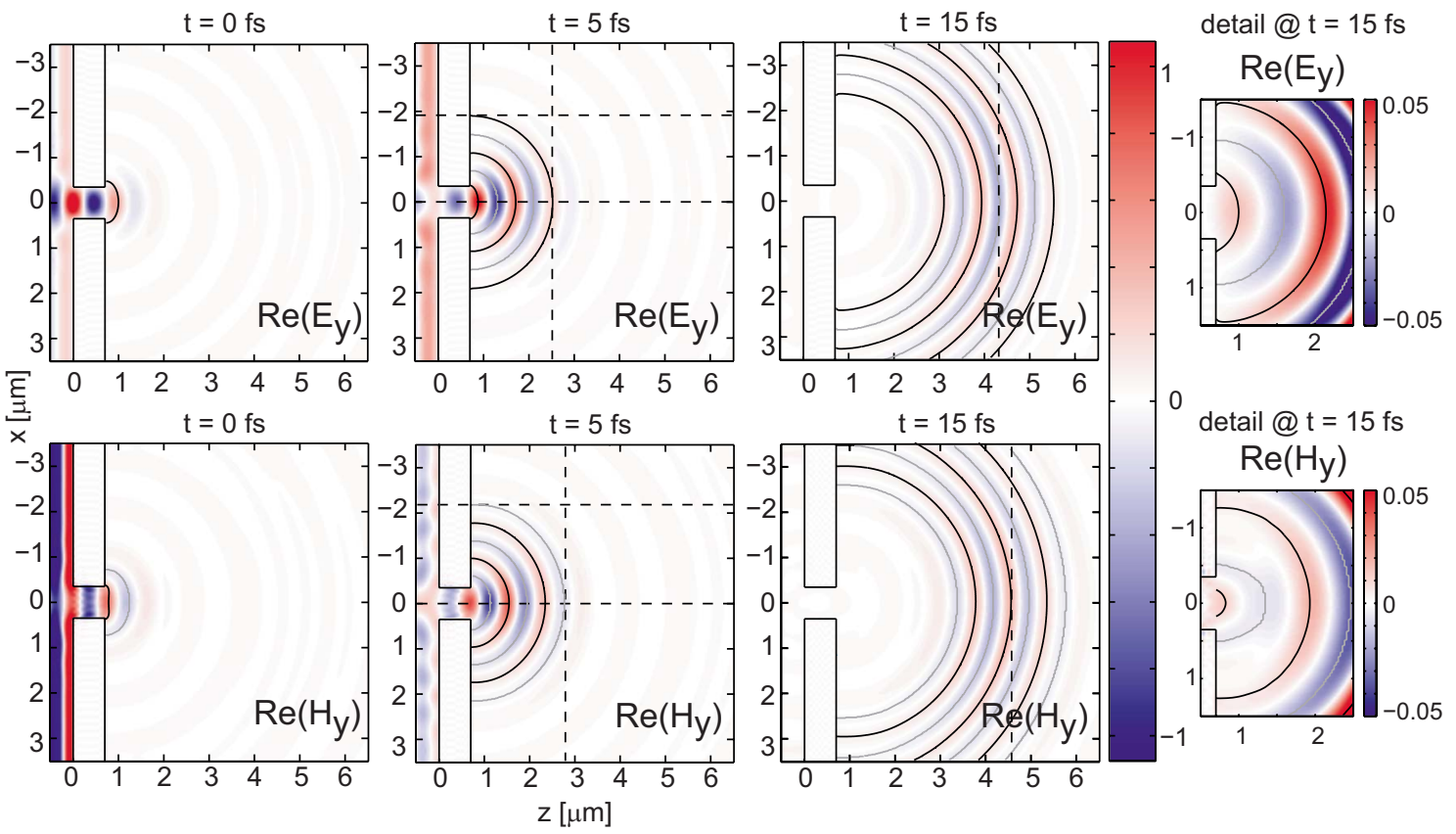

FIG. 3. (Color online) The real field for the TE (top) and TM pulse (bottom) propagating through a single-slit structure. We show the outline of the slit, contour lines to indicate the pulse maxima (black) and minima (gray), and various grid lines to guide the eye (dashed). Note, the chosen range spans only half of the amplitude range to emphasize the details of the transmitted field. The small figures on the far right show a detail of the field distribution at $t=15$ fs at a rescaled range.

boundaries. For the single-slit problem, we choose a period of $\Lambda=7.3 \mu \mathrm{m}$ and use 45 Fourier modes to represent the electric permittivity function, which is enough to ensure convergence. In the case of the double slit, the period is $\Lambda$ $=10.8 \mu \mathrm{m}$, and the electric permittivity function is represented using 65 Fourier modes.

\section{DIFFRACTION OF AN ULTRASHORT PULSE}

\section{A. Single-slit configuration}

Figure 3 presents time shots of the real field of the pulse propagating through the slit. The pulse is partially reflected by the metal layer and at $t=0$ fs the reflected earlier half of the pulse interferes with the still forward propagating trailing half. For the TE case, we expect a $\pi$-phase jump of the reflected field determined by the boundary conditions, the finite penetration depth in the aluminum in combination with the wavelength dependence of the reflection coefficient is responsible for the residual observed standing wave with a node at the metal surface. In the region in front of the slit aperture, no destructive interference occurs due to the spurious reflected field from the aluminum layer, resulting in a local maximum. The field distribution inside the slit is localized at the center and smoothly decays to zero toward the slit walls, satisfying the TE boundary conditions.

For the TM case, no phase jump is expected of the spurious reflected field, such that a standing wave with a maximum at the surface interface is created. Note that similar to the TE case, the chosen color scale spans only half of the amplitude range to emphasize the details of the transmitted field. This results in a clipping of the field in front of the slit, and therefore the local minimum at the aperture of the slit where no standing wave due to reflection occurs is less prominent. The field inside the slit is distributed more uniformly, but decays rapidly to zero inside the metal, satisfying the TM boundary conditions.

The contour lines that connect the extrema (gray for minima, black for maxima) of the field distribution shown in Fig. 3 indicate a faster propagation of the TM pulse as compared to that of the TE pulse. Also, note the reversal of the extrema for the TE pulse as compared to the TM pulse. For $t=15 \mathrm{fs}$, one can observe that the TE pulse pattern is centered at a minimum around $z=4.25 \mu \mathrm{m}$ while the TM pulse pattern is centered at a maximum around $z=4.5 \mu \mathrm{m}$. These locations are indicated by the vertical dashed grid lines at $t$ $=15$ fs in Fig. 3. Such a difference in propagated distance and pulse shape implies the presence of polarization dependent dispersion inside the slit, since the pulse travels in air everywhere else.

Another important feature is apparent when the contour lines are compared with the dashed grid lines at $t=5 \mathrm{fs}$. The contour lines are almost circular for the TE pulse propagation. However, the contour lines have a slightly elliptical shape for the TM pulse, suggesting that the wave propagating along the surface arrives earlier than the one propagating normal to the slit aperture. This effect is caused by the shape of the field distribution at the exit plane of the aperture. The TE-field distribution is highly localized, resulting in a pointlike secondary source, while the TM-field distribution is spread out uniformly, resulting in a secondary source of finite extent.

\section{Dispersion}

To explain the dispersion by the slit structure, we consider the spectral dependence of the field distribution inside the 

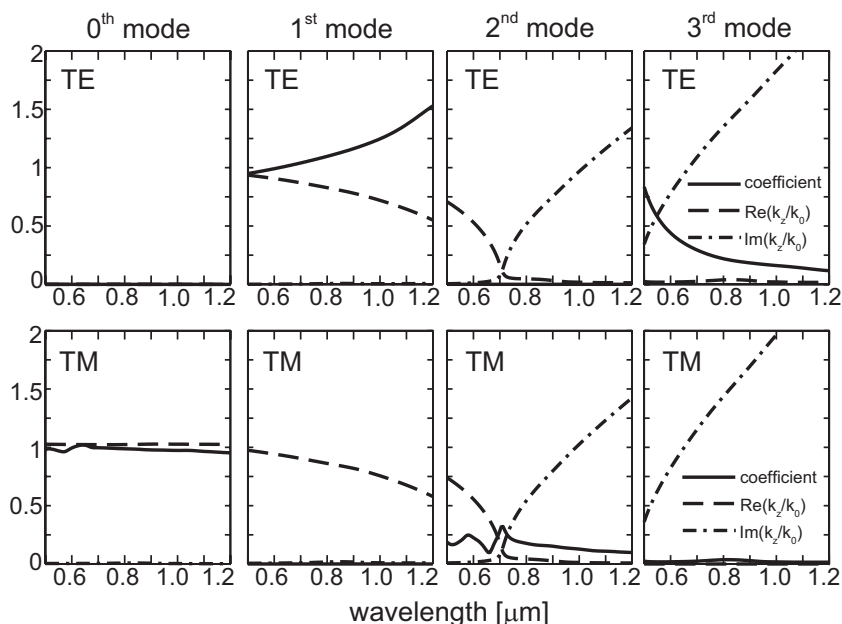

FIG. 4. The real (dashed) and imaginary part (dashed-dotted) of the propagation constant, as well as the absolute value of the coefficient (solid) as a function of the wavelength. The effective refractive index for propagating modes is proportional to $\operatorname{Re}\left(k_{z} / k_{0}\right)$.

slit, as discussed in more detail in Ref. [24]. The Fourier modal method provides the propagation constant and amplitude of each mode inside the slit that can be excited, also known as waveguide modes. In Fig. 4, we have plotted the complex propagation constant and the coefficient as a function of wavelength for the four fundamental modes. Note that the value for the propagation constant has been normalized on the wave number in vacuum $k_{0}$, which is a function of the wavelength as well. The amplitude of the coefficients has been normalized on the energy of the pulse illuminating the aperture area. The total field is obtained by a summation of the individual modes multiplied by their amplitude coefficients. The propagation constant governs the speed of propagation through the slit structure. Taking into account the modal distribution and the propagation speed of the pulse in both the $x$ and $z$ directions, the effective refractive index of the slit for propagating modes is proportional to $\operatorname{Re}\left(k_{z} / k_{0}\right)$, which is proportional to the group velocity and inversely proportional to the phase velocity.

The distribution of the TE field is dominated by the first mode, which has a transversal distribution that is comparable, but not equal, to half a period of the cosine function with its peak at the center and its nodes at the edges of the slit. Hence, the localized energy in the center of the slit for the TE pulse. Furthermore, the propagation constant (dashed line) decreases as a function of the wavelength for the first mode, yielding the dispersion relation of the TE pulse passing through the slit. Since the effective refractive index for each wavelength is smaller than for air, therefore also the group velocity is smaller than the speed of light in air; the pulse arrives later at the slit exit. The amplitude of the coefficient (solid line) increases for higher wavelengths, resulting in a redshifted field distribution. The TE pulse does not couple significantly to the second mode. A contribution from the third mode is only available as evanescent waves, with the strongest contribution close to $\lambda=0.5 \mu \mathrm{m}$, however, this spectral dependence is apodized by the weighting of the power spectrum shown in Fig. 1(a) (bottom), which is small at shorter wavelengths.
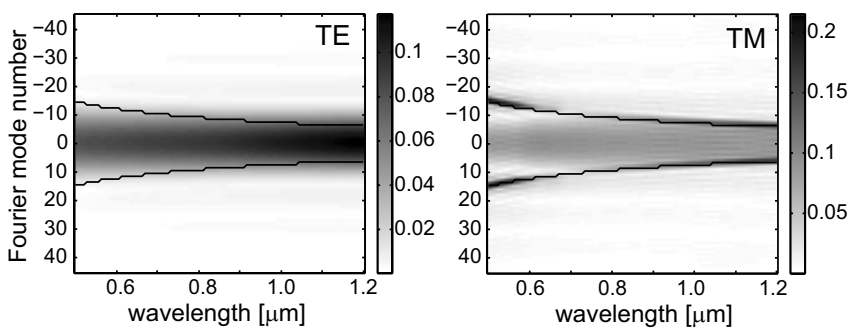

FIG. 5. Fourier mode distribution at the exit plane of the slit as a function of wavelength for TE (left) and TM illumination (right). The solid black lines indicate the transition from propagating to evanescent modes. Note the different scaling for TE and TM.

The distribution of the TM field is dominated by the zeroth mode, which has an almost constant coefficient and an effective refractive index that is close to that of air. Thus, the TM pulse propagates through the slit structure with a negligible dispersion comparable to propagation with the speed of light in air. For the second mode, the waveguide structure behaves as a low pass filter with a cutoff wavelength at $\lambda$ $\approx w$. Below this wavelength, the propagation constant decreases as function of wavelength with an oscillating amplitude of the coefficient. For $\lambda>w$, the mode becomes evanescent and the strength of the contribution depends strongly on the thickness of the metal layer. As expected, the first and the third mode do not contribute significantly since the coefficient is almost zero. Note, throughout the manuscript we implicitly scale the magnetic field component by $\left(\epsilon_{0} / \mu_{0}\right)^{1 / 2}$.

The observed time lag between the TE and TM pulse is determined by the thickness of the aluminum layer in combination with the difference of the group velocity inside the slit for both polarization states. Also, the shape of the TE pulse is determined by the dispersion relation of the propagation constant, which for an aluminum layer of $d$ $=0.7 \mu \mathrm{m}$ thickness yields a reversal of the extrema. Note that due to a partial reflection of the mode at the slit exit, a standing wave is created inside the slit which, depending on the wavelength and the thickness of the aluminum layer, determines the exact value of the coefficient, as discussed in Ref. [24].

\section{Diffraction}

The coupling of the available energy inside the slit to the free space modes is evaluated by considering a plane-wave distribution at the exit plane of the slit. In Fig. 5, we show the transmission coefficient for each Fourier mode of the TE (left) and TM pulse (right) as a function of the wavelength. The transition from propagating to evanescent modes also depends on the wavelength and is indicated by the solid lines. For modes corresponding to frequencies beyond these lines, the energy is transmitted to an evanescent surface wave. The dependence of the diffraction pattern on the wavelength can lead to spectral anomalies and spectral switches of the pulse [25].

The dominant transmission coefficients of the TE pulse are concentrated at the lower Fourier mode numbers, corresponding to a predominantly forward propagation direction in the far field. As the wavelength increases, more energy is 
(a)

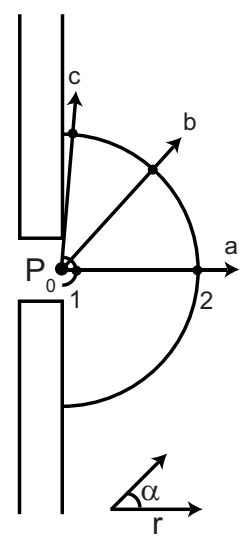

$\mathrm{r}_{1}=30 \mathrm{~nm}$

$r_{2}=3 \mu \mathrm{m}$

$\alpha_{b}=45^{\circ}$

$\alpha_{c} \sim 90^{\circ}$ (b)

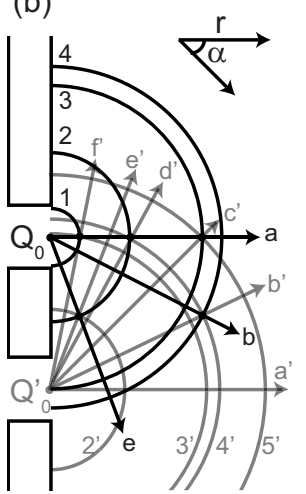

$r_{1}=0.03 \mu \mathrm{m}$

$r_{2}=1.75 \mu \mathrm{m}$

$r_{3}=3.50 \mu \mathrm{m}$

$\mathrm{r}_{4}=3.91 \mu \mathrm{m}$

$\alpha_{b}=26.6^{\circ}$

$\alpha_{\mathrm{e}} \sim 90.0^{\circ}$

FIG. 6. Definition of the observation points for (a) the singleand (b) the double-slit structure.

coupled to the evanescent modes. Therefore, a blueshift is expected for the propagating waves, and a redshift for the evanescent waves.

In the TM case, the amplitude of the transmission coefficients are more uniformly distributed over all the propagating modes. Note the different scales for both polarization states. At each wavelength, the largest contribution of the coefficients corresponds to the evanescent modes close to the $k_{0}$ boundary indicated by the solid lines, except at $\lambda \approx w$ where the slit width matches the wavelength resulting in a coupling to only propagating modes. The spectral distribution of the surface wave for a fixed slit width is similar to the discussion in Ref. [26] for a fixed wavelength but with variable slit width. At the lower side of the spectral band, where the slit width is slightly larger than a wavelength, the amplitude of the coefficients for the evanescent modes is almost twice that of the amplitude at larger wavelengths. This indicates that the surface wave excited in the TM case is dominated by the smaller wavelengths.

\section{Spatio temporal distribution}

Having discussed the dispersion and diffraction by the slit individually, now we consider the combined effect on the transmitted pulse. At four different spatial coordinates defined in Fig. 6(a), these effects are analyzed in both the spectral (left) and temporal (right) domain as shown in Fig. 7. The TE pulse is indicated by a solid line and the TM pulse by a dashed line.

The field distribution at two points on the propagation axis located at the center of the aperture $\left(P_{1 a}, P_{2 a}\right)$ show the near- and intermediate-field distribution of the ultrashort pulse propagating forward. The blueshift of the pulse is related to the dominant contribution of the shorter wavelengths for diffraction at a small angle. Since the transmission coefficients of the TE pulse at this angle are larger than that of
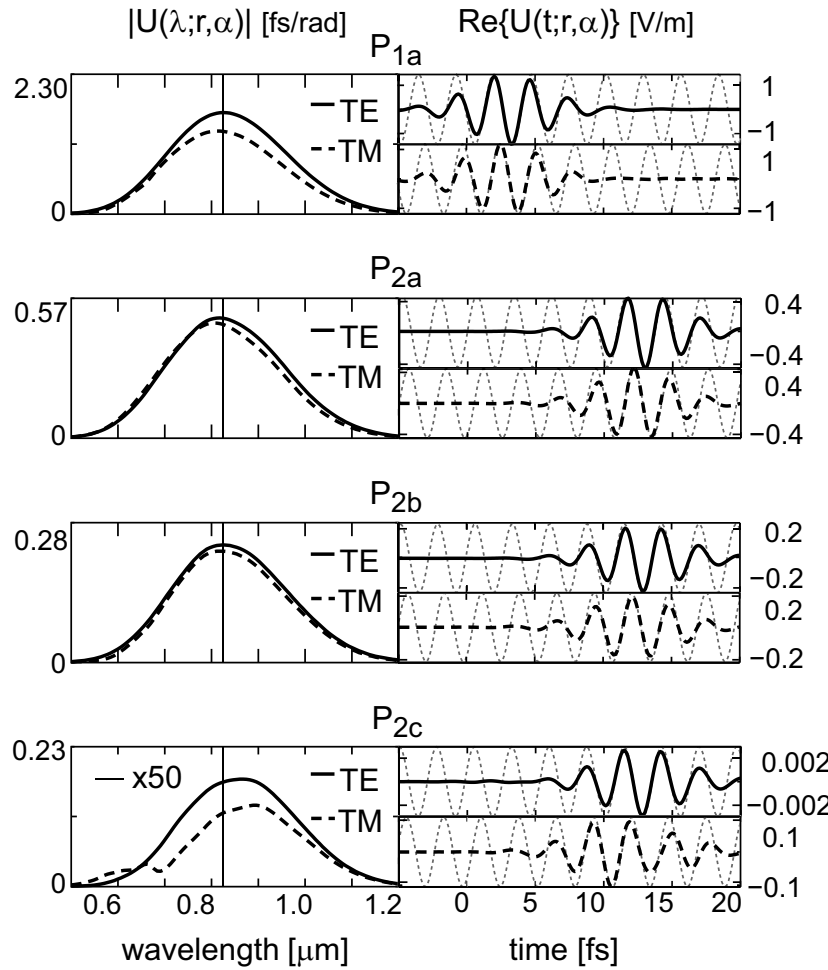

FIG. 7. The spectral (left) and temporal (right) distribution of a pulse at four distinct spatial coordinates defined in Fig. 6(a). The distribution for the TE and TM pulse are indicated by a solid and a dashed line, respectively. The vertical line in the spectral distribution indicates the peak wavelength of the original spectrum, a time trace at that wavelength is shown by the gray dotted line in the temporal distributions.

the TM pulse, the power spectrum is also higher.

The interesting phenomenon observed in Fig. 3, where the TE pulse arrives later as compared to free space propagation, and is reversed as compared to the TM pulse, is also clearly visible in the time traces of Fig. 7. The inverted sign has been experimentally observed and reported in Ref. [27]. From Sec. III A 1, we know that the effective refractive index for the first order TE mode decreases for increasing wavelength. Thus, the smallest wavelength in the spectral band travels at the highest velocity inside the waveguide, trailed by the longer wavelengths. Depending on the slit thickness, the dispersion yields a difference in optical path length for each wavelength, and consequently a pulse shape in the temporal domain for the transmitted pulse that is different from the incident pulse [24].

At point $P_{2 b}$ located at the $45^{\circ}$ diffraction angle, the spectral shape for both the TE and TM pulse is almost equal to that of the incident pulse. The initial blueshift due to the dispersion is canceled by the diffraction effects. The amplitude of the TM pulse compared to that of the TE pulse is slightly smaller over the entire spectrum.

A surface wave at a metal-air interface is a well-known phenomenon, which can only be excited by the TM pulse. Observation of the field at point $P_{2 c}$ close to the surface reveals a dip in the spectral distribution for the evanescent modes at $\lambda=w$ and a stronger contribution for $\lambda<w$ in accordance with the observations from Fig. 5. A redshift is 

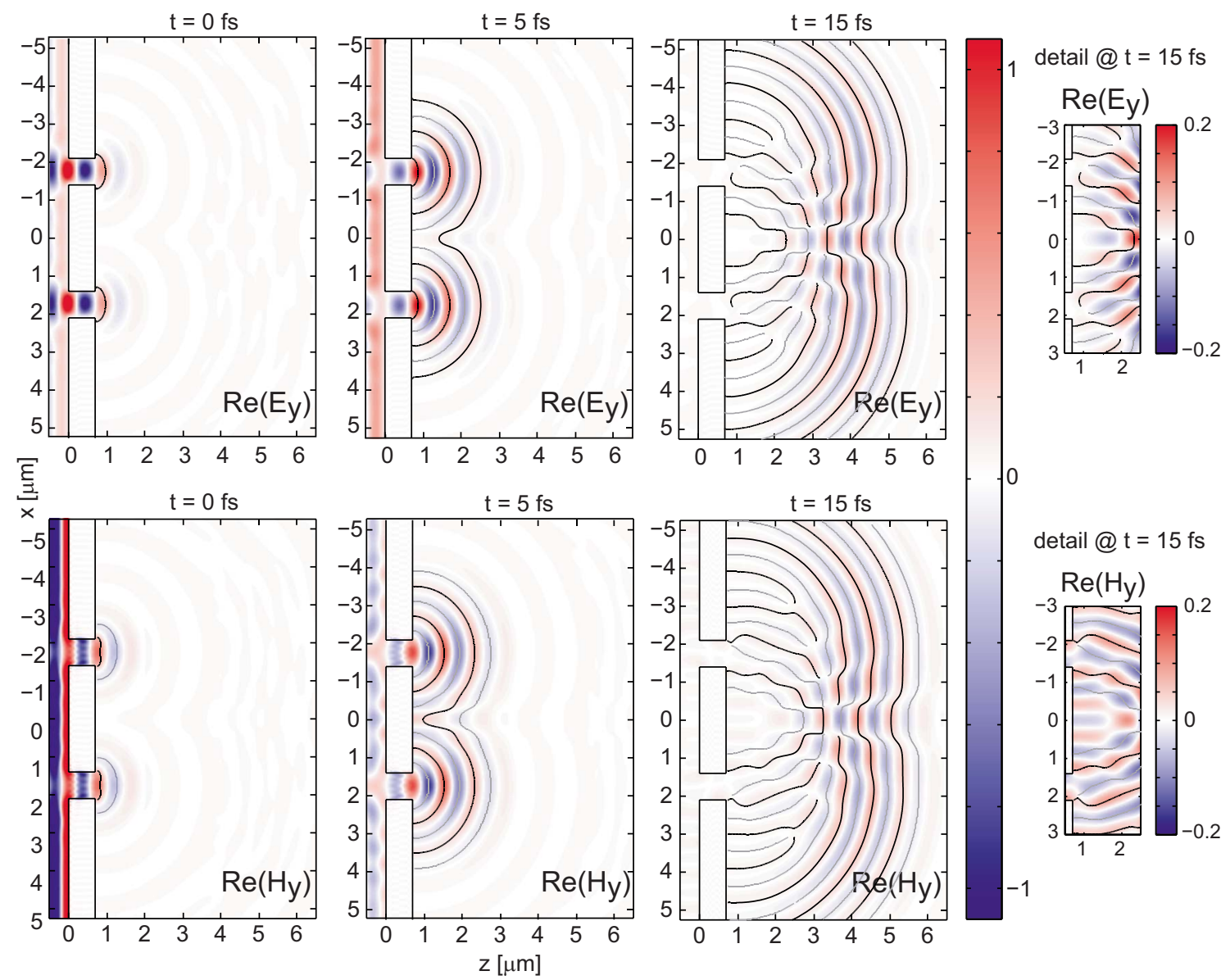

detail @ $\mathrm{t}=15 \mathrm{fs}$

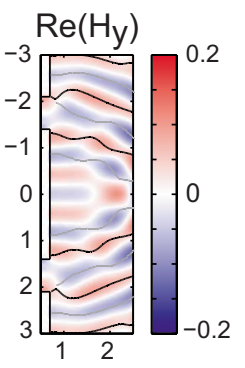

FIG. 8. (Color online) Same caption as Fig. 3 but now for the double slit and without grid lines.

observed for the TE pulse propagating close to the surface but with a considerably weaker field strength, note the $50 \times$ multiplication used to scale the amplitude of the TE pulse.

\section{B. Double-slit configuration}

Next, we study the interaction of a pulse transmitted by the single slit with that transmitted by a second slit placed at a distance $p$ from the first. This configuration is originally known as Young's double slit but now illuminated by an ultrashort pulse. Time shots of the real field of the pulse propagating through the double slit are presented in Fig. 8. Once more, the extrema are connected by black (maxima) and gray (minima) contour lines. The diffraction pattern of both slits at $t=0$ fs is similar to that of a single slit, since the distance between the two slits is chosen such that the diffracted fields cannot interact yet. Note, similarly as before, the chosen range spans only half of the amplitude range to visualize the transmitted field with more detail, but also resulting in a diminished visibility of the modulation of the reflected field for the TM pulse.

At $t=5$ fs, both diffraction patterns start to interfere. This is most clearly visible in the reflected field distribution for the TM pulse, where a more pronounced interference pattern is present close to the metal surface in contrast with Fig. 3. A standing wave with maximum modulation is created on the optic axis $(x=0)$, since the symmetry of the configuration requires that the optical path length to either slit is exactly the same. The interference of both diffraction patterns distorts the semicircular nature of the contour lines, which start to merge into a single pattern originating from the center of the two slits, as is shown at $t=15$ fs.

Furthermore, at $t=15 \mathrm{fs}$, we observe the reemission of the surface wave by the neighboring slit for the TM pulse as significantly more field is located close to the slit than for the TE pulse. This effect is clearly visible in the detail figure at $t=15$ fs on the right of Fig. 8 where the amplitude range has been rescaled. The role of surface plasmons in Young's double slit for continuous waves has been discussed in Ref. [28].

The transmission through the double slit yields similar dispersion effects as compared to the single-slit configuration, discussed in Sec. III A 1. The TM pulse is transmitted without a significant change of the pulse shape, however, the TE pulse arrives later and its pulse shape is changed, resulting in the reversal of the extrema. The Fourier distribution as a function of wavelength at the exit plane of the double slit is also highly similar to the pattern shown in Fig. 5 and discussed in Sec. III A 2. The interference between both slits results in an additional oscillation present in the pattern, which depends on the resonance condition determined by the distance between the two slits in wavelength units. Therefore, particular modes do not contribute at all while others are enhanced by a factor of 2 , depending on the wavelength. 


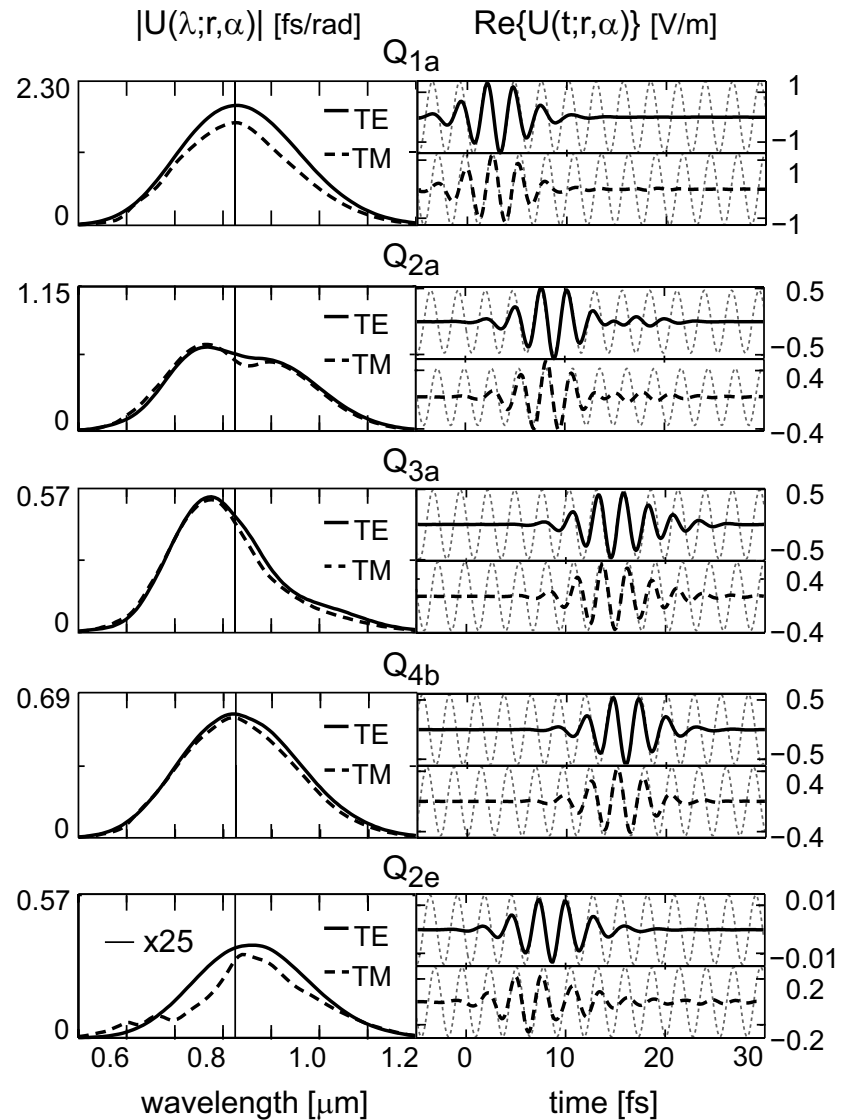

FIG. 9. Same caption as Fig. 7 but now for the five distinct spatial coordinates defined in Fig. 6(b).

The interference effects of the diffraction patterns from both slits can be clearly observed in a spatio temporal analysis at five conveniently located observation points, defined in Fig. 6(b). The points $Q$ are either located on the optic axis in the center between both slits $(x=0)$, or on the axis through the center of one of the slits $(x=1.75 \mu \mathrm{m})$. A prime $Q^{\prime}$ indicates a mirrored naming convention from the perspective of the other slit. The corresponding spectral (left) and temporal (right) distribution is presented in Fig. 9.

At point $Q_{1 a}=Q_{3 f}^{\prime}$, the spectral distribution is similar to the distribution at point $P_{1 a}$ shown in Fig. 7, since only the contribution of one of the slits is present. Equivalent to the single slit, we observe for both polarization states a blueshift of the spectrum as the distance from the slit increases. At observation point $Q_{2 a}=Q_{4 d}^{\prime}$ the spectrum of the pulse is altered severely by destructive interference at the peak wavelength, and constructive interference at $\lambda=0.75$ and $0.9 \mu \mathrm{m}$. The optical path length traversed by the pulse from the first and the second slit differs so much that we observe the pulse from the first slit being followed by the pulse from the second slit. At $Q_{3 a}=Q_{5 c}^{\prime}$ the center of the spectrum is blueshifted over a range of $50 \mathrm{~nm}$ and the width is considerably narrowed, resulting in a broadening of the associated time trace. The difference in optical path between the pulse from the first and the second slit is small enough such that the time traces overlap and consequently interference.

Due to the symmetry of the configuration, we expect a standing wave with maximum modulation on the optic axis, which simplifies the analysis for the observation points located on this axis. In the absence of near-field effects, the spectral distribution at $Q_{4 b}=Q_{4 b}^{\prime}$ has the same shape as the original pulse. Closer to the surface of the aluminum layer at $Q_{2 e}=Q_{2 e}^{\prime}$, the spectrum is redshifted. Note the $25 \times$ multiplication of the spectrum for the TE pulse. The amplitude of the field for the TM pulse is approximately twice, and for the TE pulse approximately 5 times the value of the single slit at $P_{2 c}$, due to the favorable interference condition and the shorter distance to the slit apertures.

The time trace for the TM pulse at $Q_{2 e}$ shows a continuation of the oscillations after the main pulse has passed. The surface waves are reflected at both slits, and since the spectrum of the pulse spans a broad wavelength range, it is always possible to find a wavelength at which the resonance condition between the two slits is satisfied.

\section{CONCLUSION}

We have presented a systematic study of the near and intermediate field of an ultrashort pulse transmitted by Young's double slit. First, the dispersion and diffraction effects have been discussed separately for a single slit, to gain insight in the principle phenomena determining the diffraction pattern. Next, the second slit was introduced and, when we take into account the interference effects, the resulting diffraction pattern can be explained. This has been illustrated by the observation of the time trace and spectrum of the pulse at various distinct coordinates.

Since the width of the slit is of the same dimension as the central wavelength of the pulse spectrum, the dispersion is strongly polarization dependent. The TE pulse experiences a reduced propagation speed inside the slit, while the TM pulse is virtually unaffected. The slit thickness determines the time lag and difference in pulse shape between both polarization states.

The field distribution behind the slit revealed an elliptical wave front for the TM pulse as opposed to a more circular wave front for the TE pulse, due to a uniform as opposed to a localized field distribution inside the slit, respectively. A plane wave decomposition of the light distribution transmitted by the slit shows that in the case of TE polarized light, the propagating waves are blueshifted, while evanescent waves are redshifted. Whereas for the TM polarized light, both propagating and evanescent waves are blueshifted. Moreover, at a wavelength that is equal to the slit width, the coupling to the evanescent modes is almost zero, hence no surface waves are excited.

Interference effects due to the presence of a second slit result in a highly position dependent pulse shape, corresponding to substantial spectral changes of the pulse at particular observation points in the diffraction pattern. The distance between both slits determines the resonance condition for the contribution of a particular wavelength component. At other points in the near field, the difference in traversed optical path is too large to allow any interference at all. In the center between the two slits we obtain maximum interference due to the symmetry of the configuration. In the intermediate field, the distinct diffraction patterns of both slits 
merge into one. Since the field distribution of a single slit is polarization dependent, the field distribution of the diffracted pulse in Young's double slit arrangement is also polarization dependent. The near field distribution of TM polarized light has a much stronger amplitude modulation as compared to TE polarized light due to the presence of interfering waves at the metal surface.

The strong polarization and position dependence of the near- and intermediate-field distribution of a single or double slit for illumination by an ultrashort pulse can be exploited in many near-field applications, where a highly localized control or understanding of the pulse shape is required. For example, such an understanding is critical when performing femtochemistry or spectroscopy using a near-field microscope with ultrashort pulsed light. Alternatively, switching the polarization of the incident pulse from TM to TE would give a highly sensitive means for detuning the timing and shape of the excitation pulse. For instance, this way it is possible to create circular polarized light or to switch to the complementary linear polarization, the diffraction process can be made to behave similar to a classical quarter- or halfwave plate.

Our results and observations can be verified in experiments using present technology, for example, in the microwave or terahertz wavelength ranges that allow direct measurement of amplitude and phase. The position and polarization dependence of the field distribution can be tuned by varying the width, thickness, and separation of the slits as well as by tailoring the polarization and the shape of the incident pulse. The principle of the polarization and position dependence of the diffracted pulse is not unique to slit arrangements and can be similarly observed for different types of geometries, such as circular apertures or even aperture arrays. This knowledge is essential for further investigation of more complex phenomena such as enhanced transmission of ultrafast pulsed light that involves surface plasmon generation and resonances by apertures of subwavelength dimensions.
[1] R. W. Boyd, Nonlinear Optics, 2nd ed. (Academic Press, London, 2003).

[2] Optical Imaging and Microscopy-Techniques and Advanced Systems, edited by P. Török and F.-J. Kao (Springer-Verlag, Heidelberg, 2003).

[3] U. Keller, Nature (London) 424, 831 (2003).

[4] G. Sansone et al., Science 314, 443 (2006).

[5] Z. Wang, Z. Zhang, Z. Xu, and Q. Lin, Opt. Commun. 123, 5 (1996).

[6] L. Sereda, A. Ferrari, and M. Bertolotti, J. Mod. Opt. 44, 1321 (1997).

[7] Z. Jiang, R. Jacquemin, and W. Eberhardt, Appl. Opt. 36, 4358 (1997).

[8] J. M. Anderson and C. Roychoudhuri, J. Opt. Soc. Am. A 15, 456 (1998).

[9] M. LeFrançois and S. F. Pereira, Opt. Express 11, 1114 (2003).

[10] S. P. Veetil, N. K. Viswanathan, C. Vijayan, and F. Wyrowski, Appl. Phys. Lett. 89, 041119 (2006).

[11] C. Genet and T. W. Ebbesen, Nature (London) 445, 39 (2007).

[12] H. Ichikawa, J. Opt. Soc. Am. A 16, 299 (1999).

[13] R. Müller and C. Lienau, Appl. Phys. Lett. 76, 3367 (2000).

[14] X. Ren, C. Cheng, C. Liu, H. Song, N. Zhang, and Z. Xu, Chin. Opt. Lett. 2, 63 (2004).

[15] A. J. L. Adam, J. M. Brok, A. S. van de Nes, and P. C. M. Planken, in Digest of the Joint 31st International Conference on Infrared and Millimeter Waves and 14th International Con- ference on Terahertz Electronics (Shanghai, IEEE Catalog number 06EX1385, 2006), p. 15.

[16] A. Pack, M. Hietschold, and R. Wannemacher, Ultramicroscopy 92, 251 (2002).

[17] A. Poppe, R. Holzwarth, A. Apolonski, G. Tempea, C. Spielmann, T. W. Hänsch, and F. Krausz, Appl. Phys. B: Lasers Opt. 72, 373 (2001).

[18] C. J. R. Sheppard, J. Opt. Soc. Am. A 18, 2594 (2001).

[19] C. F. R. Caron and R. M. Potvliege, J. Mod. Opt. 46, 1881 (1999).

[20] Handbook of Optical Constants of Solids, edited by E. D. Palik (Academic Press, New York, 1985).

[21] L. Li, J. Opt. Soc. Am. A 13, 1870 (1996).

[22] J. P. Hugonin and P. Lalanne, J. Opt. Soc. Am. A 22, 1844 (2005).

[23] M. Besbes et al., J. Eur. Opt. Soc. Rapid Publ. 2, 07022 (2007).

[24] A. M. Nugrowati, S. F. Pereira, and A. S. van de Nes (unpublished).

[25] J. Foley and E. Wolf, J. Opt. Soc. Am. A 19, 2510 (2002).

[26] O. T. A. Janssen, H. P. Urbach, and G. W. 't Hooft, Opt. Express 14, 11823 (2006).

[27] H. Cao and A. Nahata, Opt. Express 12, 3664 (2004).

[28] H. F. Schouten, N. Kuzmin, G. Dubois, T. D. Visser, G. Gbur, P. F. A. Alkemade, H. Blok, G. W. 't Hooft, D. Lenstra, and E. R. Eliel, Phys. Rev. Lett. 94, 053901 (2005). 